\title{
STEADY GENERAL RELATIVISTIC MAGNETOHYDRODYNAMIC INFLOW/OUTFLOW SOLUTION ALONG LARGE-SCALE MAGNETIC FIELDS THAT THREAD A ROTATING BLACK HOLE
}

\author{
Hung-Yi Pu ${ }^{1}$, Masanori Nakamura ${ }^{1}$, Kouichi Hirotani ${ }^{1}$, Yosuke Mizuno ${ }^{2,3}$, Kinwah Wu ${ }^{4}$, and Keilchi Asada ${ }^{1}$ \\ ${ }^{1}$ Institute of Astronomy \& Astrophysics, Academia Sinica, 11F of Astronomy-Mathematics Building, AS/NTU No. 1, Taipei 10617, Taiwan \\ ${ }^{2}$ Institute of Astronomy, National Tsing Hua University, Hsinchu 30013, Taiwan \\ ${ }^{3}$ Institute for Theoretical Physics, Goethe University, D-60438 Frankfurt am Main, Germany \\ ${ }^{4}$ Mullard Space Science Laboratory, University of College London, Holmbury St Mary, Dorking, Surrey RH5 6NT, UK \\ Received 2014 August 25; accepted 2015 January 8; published 2015 March 4
}

\begin{abstract}
General relativistic magnetohydrodynamic (GRMHD) flows along magnetic fields threading a black hole can be divided into inflow and outflow parts, according to the result of the competition between the black hole gravity and magneto-centrifugal forces along the field line. Here we present the first self-consistent, semi-analytical solution for a cold, Poynting flux-dominated (PFD) GRMHD flow, which passes all four critical (inner and outer, Alfvén, and fast magnetosonic) points along a parabolic streamline. By assuming that the dominating (electromagnetic) component of the energy flux per flux tube is conserved at the surface where the inflow and outflow are separated, the outflow part of the solution can be constrained by the inflow part. The semi-analytical method can provide fiducial and complementary solutions for GRMHD simulations around the rotating black hole, given that the black hole spin, global streamline, and magnetizaion (i.e., a mass loading at the inflow/outflow separation) are prescribed. For reference, we demonstrate a self-consistent result with the work by McKinney in a quantitative level.
\end{abstract}

Key words: black hole physics - galaxies: active - galaxies: jets - magnetohydrodynamics (MHD)

\section{INTRODUCTION}

Relativistic jets emerging from accreting black hole systems have been observed in active galactic nuclei (AGNs), microquasars (stellar mass black hole X-ray binaries), and presumably gamma-ray bursts (GRBs). Observationally, the bulk Lorentz factors $\Gamma$ of jets in AGNs are $\gtrsim 10-20$ (Jorstad et al. 2005; Cohen et al. 2007; Gu et al. 2009; Pushkarev et al. 2009; Lister et al. 2013), and the values could be higher for some blazars (see Krishna et al. 2006; Hovatta et al. 2009). The Lorentz factors of micro-quasar jets are lower, mostly with $\Gamma \sim 2-10$ (e.g., Corbel et al. 2002; Fender et al. 2004), but still there are a few found to have $\Gamma>10$ (see Miller-Jones et al. 2006). Jets in gamma-ray bursters are supposed to be ultra-relativistic, and their Lorentz factors can be as high as $\sim 100$ - 1000 (see, e.g., Lyubarsky 2010; Lyutikov 2011). How jets become relativistic after being launched from nearby black holes is a long-standing issue. Electromagnetic or magnetohydrodynamic (MHD) mechanisms are frequently invoked to extract energy and momentum from the black hole and accretion disk (e.g., Meier et al. 2001, for reviews). One of the key issues to be addressed is the potential of the MHD flow acceleration up to a high bulk Lorentz factor of $\Gamma>10$.

An ideal engine to power relativistic jets is a spinning black hole. Close to the black hole, the rapid winding of the azimuthal component in large-scale magnetic fields due to the frame-dragging inside the black hole ergosphere results in a counter torque (induced by the Lorentz force) against a black hole rotation. The energy that the black hole spent to perturb the field line can be propagated outward in the form of torsional Alfvén waves, thus extracting the black hole energy electromagnetically (Blandford \& Znajek 1977, hereafter BZ77). However, because the environment around an accreting black hole is not a perfect vacuum (contrary to the force-free treatment in BZ77), the general relativistic magnetohydrodynamics (GRMHD), which consist of electromagnetic and fluid components, provide a more general picture for the dynamics and structures of relativistic jets in both theoretical approaches (e.g., Camenzind 1986a, 1986b, 1987; Takahashi et al. 1990; Fendt \& Camenzind 1996; Fendt \& Greiner 2001; Fendt \& Ouyed 2004) and numerical simulations (e.g., Koide et al. 1998, 2000; Mizuno et al. 2004; McKinney \& Gammie 2004; Hawley \& Krolik 2006; McKinney 2006; Beckwith et al. 2008; Tchekhovskoy et al. 2010, 2011; Tchekhovskoy \& McKinney 2012).

The overall configuration of an accreting black hole system is schematically illustrated in Figure 1 (see also GRMHD simulations for magnetized accretion, e.g., McKinney 2006; McKinney \& Gammie 2004; Hawley \& Krolik 2006). Ordered, parabolic lines are developed near the funnel, which is confined by the corona and/or accretion flow. Due to the relative absence of accreting materials, the funnel region is Poynting fluxdominated (PFD). The fluid loading onto the field is accelerated inward (or outward) if the black hole gravity force is larger (or smaller) than the "magneto-centrifugal" forces (e.g., Sagdowski \& Sikora 2010, in the case of the accretion disk). As pointed out in the theoretical work of Takahashi et al. (1990), black hole rotational energy can be extracted outward by a PFD GRMHD inflow. This is a direct result of the electromagnetic components dominating the GRMHD flow, and the electromagnetic component is responsible for extracting the black hole energy, similar to the BZ77 process. The outward energy flux, after being extracted from the black hole, is expected to propagate continuously outward throughout the magnetosphere from the inflow region to the outflow one. In this paper, we focus on the PFD GRMHD flow in the funnel region, including both the inflow and outflow parts.

For comparison, let us quickly consider the case when the GRMHD flow becomes fluid-dominated. In that case, the energy flux is dominated by the fluid component, and therefore has an inward direction for inflow, but outward for outflow (see 


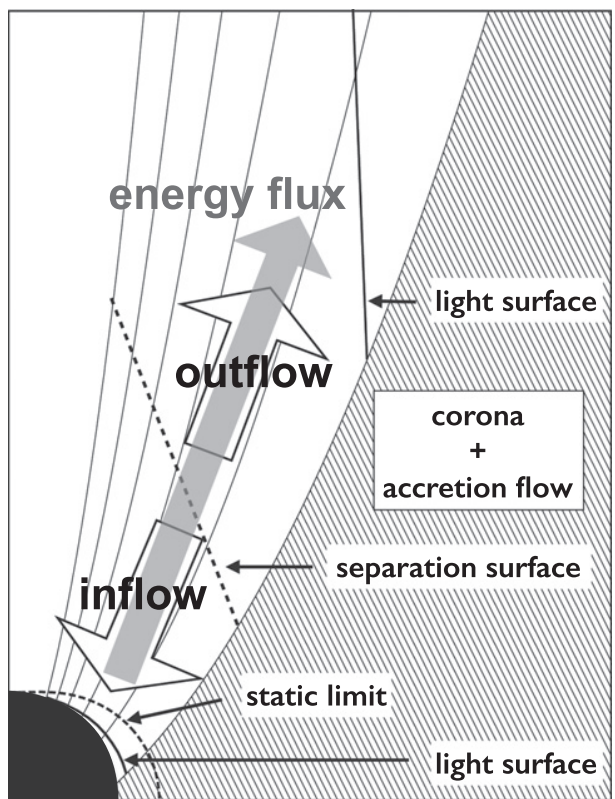

Figure 1. Schematic illustration of a Poynting flux-dominated (PFD) GRMHD flow confined by the accretion flow and its corona. The outward-streaming curves indicates ordered, large-scale magnetic fields that thread the black hole event horizon. The inflows and the outflows (represented by thick white arrows) are along the field lines, and are separated by the separation surface (marked by a dashed line). The energy flux (represented by a gray arrow) is outward in both the inflow and outflow regions, as the black hole rotational energy is extracted and transported outward. The static limit (dashed curve) and the light surface (solid curve) outside the black hole (black region) are also shown.

the energy flux direction shown in Figure 1). Such discontinuity of the energy and momentum fluxes implies that the outflow is accretion-powered, which is constrained by the energy input from the disk/corona. The switch-on and switchoff of the extraction of the black hole energy (inflow) may closely relate to the launching and quenching of relativistic jets (outflow) (e.g., Pu et al. 2012; Globus \& Levinson 2013).

Prior to the GMRHD studies mentioned, Phinney (1983) considered the inflow and outflow along a monopole field jointly by the conservation of the total energy flux per flux tube. In this pioneering work, they consider energy extraction from the black hole via BZ77 process (the inflow part), and the Michel's "minimum torque solution" (Michel 1969), in which the fast(-magnetosonic) point is located at infinity (the outflow part). We, however, suggest that a more realistic situation can be considered: the black hole energy extraction process in the framework of GRMHD, and a type of parabolic GRMHD flows as a result of external pressure confinements provided by the corona/accretion. Recent observational evidence also supports this idea; nearby active radio galaxy, M87, exhibits the parabolic streamline up to $\sim 10^{5}$ Schwarzschild radius (Asada \& Nakamura 2012).

Furthermore, we are interested in the case that the fast point of the outflow is located at a finite distance. This consideration is directly related the conversion from Poynting to kinetic energy fluxes of the flow and therefore the jet acceleration. Poloidal magnetic flux is required to diverge sufficiently rapidly in order for most of the Poynting flux to be converted into the kinetic energy flux beyond the fast point (also known as the magnetic nozzle effect; e.g., (Camenzind 1989; Li et al. 1992; Begelman \& Li 1994; Takahashi \& Shibata 1998).
Beskin \& Nokhrina (2006) examine the acceleration of the jet along a parabolic streamline by introducing a small perturbation into the force-free field. As a result, the fast point is located at a finite distance. This indicates how plasma loading in the flow plays a role in accelerating the flow, as well as a conversion from Poynting to kinetic/particle energies. They consider the behavior of the outflow in the flat spacetime. However, we are interested in both the inflow and outflow near a black hole.

All of these theoretical works provide important pieces toward a picture that includes the following process along the field line: (i) in the inflow region the rotational energy of the black hole is extracted outward by the GRMHD inflow, (ii) at the the inflow/outflow separation surface the extracted energy flux is carried out continuously, and (iii) in the outflow region the flow passes the fast point, and hence the bulk Lorentz factor increases. Although this picture has already been recognized in the quasi-steady state in GRMHD simulations (e.g., McKinney \& Gammie 2004; Hawley \& Krolik 2006; McKinney 2006), no steady solution is available in the literature.

In this paper, we present the first semi-analytical work. We consider the energy extraction from the black hole via the GRMHD (inflow), and the perturbed force-free parabolic field line in Beskin \& Nokhrina (2006) (outflow). With given black hole spin, field angular velocity, and magnetization at the separation surface, we are able to to constrain the outflow solution by the inflow solution. For reference, we adopt similar parameters reported in the GRMHD simulation of McKinney (2006; hereafter M06). Our semi-analytical solution passes all the critical points (inner and outer, Alfvén, and fast points), and agrees with the inflow and outflow properties along a mid-level field line in M06.

The paper is organized as follows. In Section 2, we outline the GRMHD formulation and the wind equation (WE). In Section 3, with the consideration of the conservation of energy flux in inflow and outflow region near the separation surface, we discuss the matching condition to connect the inflow and outflow part of a PFD GRMHD flow. In Section 4, we introduce our model setup. We adopt similar parameters to those reported by M06, and compare the solution obtained by the matching condition with that of the time-averaged GRMHD numerical simulation results in M06. Finally, a summary is given in Section 5.

\section{STATIONARY AXISYMMETRIC MHD FLOW IN A KERR SPACETIME}

\subsection{Basic Formulae}

The theory about stationary and axisymmetric ideal GRMHD flows has been in several works (Camenzind 1986a, 1986b, 1987; Takahashi et al. 1990; Fendt \& Camenzind 1996; Fendt \& Greiner 2001; Fendt \& Ouyed 2004). For completeness, in this section we summarize and present the necessary formulae for this paper.

The natural unit system is used throughout this work. As $c=G=M=1$, the gravitational radius $r_{\mathrm{g}}=G M / c^{2}=1$, where $c$ is the speed of light, $G$ is the gravitational constant, and $M$ is the mass of the black hole (conversions from the c.g. s. units to the natural units for the physical variables here can be found in Tables 3 and 4 in Pu et al. 2012). The flows occur in a background Kerr spacetime, which is stationary and axisymmetric. For a metric signature $[-+++]$, the Kerr 
metric (in Boyer-Lindquist coordinates) reads

$$
\begin{aligned}
d s^{2}= & -\frac{\Delta-a^{2} \sin ^{2} \theta}{\Sigma} d t^{2}-\frac{4 a r \sin ^{2} \theta}{\Sigma} d t d \phi \\
& +\frac{A \sin ^{2} \theta}{\Sigma} d \phi^{2}+\frac{\Sigma}{\Delta} d r^{2}+\Sigma d \theta^{2},
\end{aligned}
$$

where $a \equiv J$ is the angular momentum of the black hole, $\Delta \equiv r^{2}-2 r+a^{2}, \quad \Sigma \equiv r^{2}+a^{2} \cos ^{2} \theta, \quad$ and $A \equiv\left(r^{2}-a^{2}\right)^{2}+\triangle a^{2} \sin ^{2} \theta$.

We also assume that the flow is cold. For a highly-relativistic flow, the thermal pressure $p$ is insignificant compared with the rest-mass energy density and the kinetic energy density in the fluid, and hence the cold limit is justified.

The flow is magnetized and the stress-energy tensor of the fluid has two components:

$$
T^{\mu \nu}=T_{\mathrm{FL}}^{\mu \nu}+T_{\mathrm{EM}}^{\mu \nu},
$$

where the fluid component is given by

$$
T_{\mathrm{FL}}^{\mu \nu}=\rho u^{\mu} u^{\nu},
$$

and the electromagnetic component by

$$
T_{\mathrm{EM}}^{\mu \nu}=\frac{1}{4 \pi}\left(F^{\mu \gamma} F_{\gamma}^{\nu}-\frac{1}{4} g^{\mu \nu} F^{\alpha \beta} F_{\alpha \beta}\right),
$$

where $u^{\mu}$ is the four-velocity of the fluid and $\rho$ is the rest-mass energy density. The electromagnetic field tensor $F_{\mu \nu}$ satisfies Maxwell's equations, and the proper number density $n(=\rho / m$; where $m$ is the rest-mass of the particles) satisfies the mass continuity equation.

Under the ideal MHD condition, a stationary and axisymmetric flow obeys four conservation laws:

$$
\begin{gathered}
\left(n u^{\mu}\right)_{; \mu}=0 \\
F_{\mu \nu} u^{\mu}=0 \\
\left(\xi_{\mu} T^{\mu \nu}\right)_{; \nu}=0 \\
\left(\eta_{\mu} T^{\mu \nu}\right)_{; \nu}=0
\end{gathered}
$$

where $\xi^{\mu}=\partial / \partial t$ and $\eta^{\mu}=\partial / \partial \phi$ are the Killing vectors. These conservation equations (Equations (5)-(8)) give four conserved quantities along a streamline. By denoting the poloidal stream function as $\Psi(r, \theta)$, they are: (i) the angular velocity of the field line, $\Omega_{F}(\Psi)$; (ii) the particle number flux per unit magnetic flux (mass loading), $\eta(\Psi)$; (iii) the total energy of the flow per particle, $E(\Psi)$; and (iv) the total angular momentum per particle, $L(\Psi)$ (Camenzind 1986a, 1986b, 1987; Takahashi et al. 1990):

$$
\begin{gathered}
\Omega_{F}(\Psi)=\frac{F_{t r}}{F_{r \phi}}=\frac{F_{t \theta}}{F_{\theta \phi}} \\
\eta(\Psi)=\frac{\sqrt{-g} n u^{r}}{F_{\theta \phi}}=-\frac{\sqrt{-g} n u^{\theta}}{F_{r \phi}} \\
=\frac{\sqrt{-g} n u^{t}\left(\Omega-\Omega_{F}\right)}{F_{r \theta}}
\end{gathered}
$$

$$
\begin{aligned}
E(\Psi) & =E_{\mathrm{FL}}+E_{\mathrm{EM}} \\
& =-\mu u_{t}-\frac{\Omega_{F}}{4 \pi \eta} \sqrt{-g} F^{r \theta} \\
& =-\mu u_{t}-\frac{\Omega_{F}}{4 \pi \eta} B_{\phi}, \\
L(\Psi) & =L_{\mathrm{FL}}+L_{\mathrm{EM}} \\
& =\mu u_{\phi}-\frac{1}{4 \pi \eta} \sqrt{-g} F^{r \theta} \\
& =\mu u_{\phi}-\frac{B_{\phi}}{4 \pi \eta},
\end{aligned}
$$

with $\sqrt{-g}=\Sigma \sin \theta$. Here, $\Omega=u^{\phi} / u^{t}$ is the fluid angular velocity and $\mu$ is the relativistic specific enthalpy, which becomes $m$ in the cold limit. The covariant magnetic field observed by a distant observer with $u^{\nu}=(1,0,0,0)$ is given by

$$
B_{\mu} \equiv \frac{1}{2} \epsilon_{\nu \mu \alpha \beta} F^{\alpha \beta} u^{\nu}
$$

and its toroidal component is given by

$$
B_{\phi}=\sqrt{-g} F^{r \theta}=(\Delta / \Sigma) \sin \theta F_{r \theta},
$$

where $\epsilon_{\nu \mu \alpha \beta}=\sqrt{-g}[\nu \mu \alpha \beta]$ is the Levi-Civita tensor, and $[\nu \mu \alpha \beta]$ is the completely antisymmetric symbol (see the Appendix).

The outward energy flux in the flow is

$$
\mathcal{E}^{r}=-T_{t}^{r}=n E u^{r},
$$

and the outward angular momentum flux is

$$
\mathcal{L}^{r}=-T_{\phi}^{r}=n L u^{r} .
$$

Splitting them into fluid (i.e., $E_{\mathrm{FL}}$ and $L_{\mathrm{FL}}$ ) and the electromagnetic components (i.e., $E_{\mathrm{EM}}$ and $L_{\mathrm{EM}}$ ) gives

$$
\begin{aligned}
\mathcal{E}^{r} & =\mathcal{E}_{\mathrm{FL}}^{r}+\mathcal{E}_{\mathrm{EM}}^{r} \\
& =n E_{\mathrm{FL}} u^{r}+n E_{\mathrm{EM}} u^{r} \\
& =-n \mu u_{t} u^{r}-\frac{\Omega_{F}}{4 \pi} \frac{B_{\phi}}{\Sigma \sin \theta} A_{\phi, \theta},
\end{aligned}
$$

and

$$
\begin{aligned}
\mathcal{L}^{r} & =\mathcal{L}_{\mathrm{FL}}^{r}+\mathcal{L}_{\mathrm{EM}}^{r} \\
& =n L_{\mathrm{FL}} u^{r}+n L_{\mathrm{EM}} u^{r} \\
& =-n \mu u_{\phi} u^{r}+\frac{\mathcal{E}_{\mathrm{EM}}^{r}}{\Omega_{F}} .
\end{aligned}
$$

As initially proposed by Takahashi et al. (1990), the case of $\mathcal{E}^{r}>0$ for inflow (which requires a negative total energy) is known as the MHD Penrose process. For later studies, the term is instead used to indicate a negative energy orbit of the fluid component, $\mathcal{E}_{\mathrm{FL}}^{r}>0$ (e.g., Hirotani et al. 1992; Koide et al. 2002; Semenov et al. 2004; Komissarov 2005; Koide \& Baba 2014).

The bulk Lorentz factor of the flow for a distinct observer can be defined by

$$
\Gamma=\sqrt{-g_{t t}} u^{t}
$$

If all the energy in the Ponyting flux is converted to the fluid's bulk (kinetic) energy at a large distance, the terminal Lorentz 
factor will be

$$
\Gamma_{\infty}=\frac{E}{\mu}=\frac{\mathcal{E}^{r}}{\rho u^{r}}
$$

In addition, the angular velocity of the fluid at a large distance will be

$$
u_{\infty}^{\phi}=\frac{L}{\mu}=\frac{\mathcal{L}^{r}}{\rho u^{r}}
$$

Equations (20) and (21) therefore provide the upper limit of the terminal Lorentz factor and angular velocity of the fluid at large distances.

\subsection{Wind Equation}

The streamline of the flow is represented by the function $\Psi(r, \theta)=$ const. The WE (i.e., the relativistic Bernoulli equation) describing the fluid motion along the streamlines can be obtained using the normalization condition $u^{\alpha} u_{\alpha}=-1$. The WE therefore has the form:

$$
u_{p}^{2}+1=\left(\frac{E}{\mu}\right)^{2} U_{g}(r, \theta),
$$

where the poloidal component of the four-velocity is given by

$$
u_{p}^{2}=u^{j} u_{j}
$$

with the summation over the poloidal indices $j=\{r, \theta\}$. The term $U_{g}(r, \theta)$ in the right-hand side of Equation (22), which is evaluated along the magnetic field line in the calculation, is related to the conserved quantities, and its explicit expression depends on the assumed background spacetime (see Camenzind 1986a, 1986b, 1987; Fendt \& Camenzind 1996; Fendt \& Ouyed 2004 for the Minkowski and Schwarzschild spacetimes; and Takahashi et al. (1990); Fendt \& Greiner (2001) for the Kerr spacetime).

In a Kerr spacetime, we obtain

$$
U_{g}(r, \theta)=\frac{K_{0} K_{2}-2 K_{2} M_{A}^{2}-K_{4} M_{A}^{4}}{\left(M_{A}^{2}-K_{0}\right)^{2}},
$$

(Takahashi et al. 1990), where

$$
\begin{gathered}
K_{0}=-\left(g_{\phi \phi} \Omega_{F}^{2}+2 g_{t \phi} \Omega_{F}+g_{t t}\right), \\
K_{2}=\left(1-\Omega_{F} \frac{L}{E}\right)^{2}, \\
K_{4}=\frac{-1}{g_{t \phi}^{2}-g_{t t} g_{\phi \phi}}\left[g_{\phi \phi}+2 g_{t \phi} \frac{L}{E}+g_{t t}\left(\frac{L}{E}\right)^{2}\right] .
\end{gathered}
$$

The Alfvén Mach number $M_{A}$ is given by

$$
M_{A}^{2} \equiv 4 \pi \mu \eta^{2} / n=4 \pi \mu n\left(\frac{u_{p}}{B_{p}}\right)^{2}=4 \pi \mu \eta\left(\frac{u_{p}}{B_{p}}\right),
$$

where the re-scaled poloidal field is

$$
\begin{aligned}
B_{p}^{2} & =B^{j} B_{j}\left(g_{t t}+g_{t \phi} \Omega_{F}\right)^{-2} \\
& =\frac{1}{\Delta \sin ^{2} \theta}\left[g^{r r} \Psi_{, r}^{2}+g^{\theta \theta} \Psi_{, \theta}^{2}\right] .
\end{aligned}
$$

Along the streamline several characteristic surfaces can be defined. Their definition and properties are summarized in the Appendix.

The conserved quantities $E, L$, and $\eta$ can be expressed in terms of three system parameters: (i) the launching point of the flow, $r_{\star}$; (ii) the location of the Alfvén surface, $r_{A}$; and (iii) the magnetization parameter at the launching point, $\sigma_{\star}$. Explicitly, the relations are

$$
\begin{gathered}
\frac{L}{E}=-\left.\frac{g_{t \phi}+g_{\phi \phi} \Omega_{F}}{g_{t t}+g_{t \phi} \Omega_{F}}\right|_{r_{A}}, \\
\tilde{E}^{2}=\left.\frac{K_{0}}{K_{2}}\right|_{r_{\star}}, \\
\sigma_{\star}=\frac{\Phi_{\star}^{2}}{\left.4 \pi m\left(n u_{p} \sqrt{-g}\right)\right|_{r_{\star}}}=\frac{\Phi_{\star}}{4 \pi m} \frac{1}{|\eta|},
\end{gathered}
$$

where $\tilde{E}=E / \mu$, the flux function $\Phi=B_{p} \sqrt{-g}$, and $\Phi_{\star}$ denotes that it is evaluated at $r=r_{\star}$. In terms of these parameters, the Mach number can be written as

$$
M_{A}=\left.\left[\frac{\mu}{m} u_{p} \sqrt{-g} \frac{f}{\sigma_{\star}}\right]\right|^{1 / 2},
$$

where $f=\Phi_{\star} / \Phi$. Note that $u_{p}=0$ at $r=r_{\star}$ has been assumed in deriving the relation (31). In addition, the relation (32) implies that knowing the mass loading $\eta$ is equivalent to knowing the $\sigma_{\star}$.

In the cold limit, the WE is a polynomial equation of 4th order in $u_{p}$ :

$$
\sum_{i=0}^{4} \mathcal{A}_{i} u_{p}^{i}=0
$$

The coefficients $\mathcal{A}_{i}\left(r ; \Psi, \Phi, r_{\star}, r_{A}, \sigma_{\star}, \Omega_{F}\right)$ are given by

$$
\begin{aligned}
& \mathcal{A}_{4}=C_{2}^{2}, \\
& \mathcal{A}_{3}=-2 C_{2} K_{0}, \\
& \mathcal{A}_{2}=C_{2}^{2}+K_{0}^{2}+\tilde{E}^{2} K_{4} C_{2}^{2} \\
& \mathcal{A}_{1}=-2 C_{2} K_{0}+2 \tilde{E}^{2} K_{2} C_{2} \\
& \mathcal{A}_{0}=K_{0}^{2}-\tilde{E}^{2} K_{0} K_{2},
\end{aligned}
$$

where $C_{2}=\sqrt{-g} f / \sigma_{\star}($ Fendt \& Greiner 2001).

\section{MATCHING CONDITION OF THE INFLOW AND OUTFLOW}

In the work of Phinney (1983), the matching of the inflow and outflow parts of the flow is constrained by the conservation of the energy flux per magnetic flux in the inflow and outflow region

$$
(\eta E)_{\text {inflow }}=(\eta E)_{\text {outflow }} \text {. }
$$


Remember that $\eta$ and $E$ of the inflow and outflow are both constant.

Consider Equation (35) at the separation surface, $r_{s}$, for PFD flow $\left(E \approx E_{\mathrm{EM}} \gg E_{\mathrm{FL}}\right)$, we further consider

$$
(\eta)_{\text {inflow }}\left(E_{\mathrm{EM}}\right)^{-}=(\eta)_{\text {outflow }}\left(E_{\mathrm{EM}}\right)^{+},
$$

to be the matching condition of the inflow and outflow. The superscripts "-" (or "+"), respectively, denote the physical value computed at the location very close to $r_{s}$ in the inflow (or outflow) region, that is, $r \rightarrow r_{s}^{-}$(or $r \rightarrow r_{s}^{+}$). After some algebra, Equation (36) can also be expressed as

$$
\left(\mathcal{E}_{\mathrm{EM}}^{r}\right)^{-}=\left(\mathcal{E}_{\mathrm{EM}}^{r}\right)^{+},
$$

or

$$
\frac{\Omega_{F}}{4 \pi}\left(B_{\phi}\right)^{-}=\frac{\Omega_{F}}{4 \pi}\left(B_{\phi}\right)^{+} .
$$

Equation (37) implies that the matching condition we adopt is equivalent to the statement: the outward Poyting energy flux is continuous at the separation surface. ${ }^{5}$ Equation (38) reveals that such condition guarantees that the toroidal field is continuous at the separation point, provided that $\Omega_{F}$ is the same constant in the inflow and outflow region.

It is interesting to note that the matching condition does not require that $\eta$ or $E_{\mathrm{EM}}$ should be continuous when crossing $r=r_{s}$. That is, if we define

$$
\delta \equiv\left|\frac{(\eta)_{\text {inflow }}}{(\eta)_{\text {outflow }}}\right|=\left|\frac{\left(E_{\mathrm{EM}}\right)^{+}}{\left(E_{\mathrm{EM}}\right)^{-}}\right|=\left|\frac{\left(\sigma_{\star}\right)^{+}}{\left(\sigma_{\star}\right)^{-}}\right|,
$$

$\delta$ is not necessary for unity. The last relation in Equation (39) is obtained with the help of Equation (32). Nevertheless, the outflow can still be properly constrained by the inflow even with the uncertainty of $\delta$.

Consider a flow along a prescribed, hole-threading poloidal field line with some specific angular velocity field. Znajek (1977) showed that, due to the regularity requirement at the event horizon, $r_{+}=1+\sqrt{1-a^{2}}$, the derivative of the stream function $\Psi$ is finite and $B_{\phi}$ satisfies

$$
B_{\phi}\left(r_{+}, \theta\right)=-\sin \theta \frac{r_{+}^{2}+a^{2}}{r_{+}^{2}+a^{2} \cos ^{2} \theta}\left(\Omega_{H}-\Omega_{F}\right) \Psi_{, \theta},
$$

where $\Omega_{H}$ is the angular velocity of the hole. As a result, $\left(B_{\phi}\right)^{-}$ is insensitive to different value of $\left(\sigma_{\star}\right)^{-}\left(\left(\boldsymbol{B}_{\phi}\right)^{-} \approx\right.$ const.). From dynamical point of view, this can be understood by the fact that the fast point of a PFD GRMHD inflow is always located close to the black hole event horizon (Appendix).

For outflow, however, there is no constraint at infinity, and therefore $\left(B_{\phi}\right)^{+}$depends on $\left(\sigma_{\star}\right)^{+}$more strongly. Again, from a dynamical point of view, the relatively strong dependence can be understood by the fact that the fast point of the outflow can vary from finite distance to infinity. Because the uncertainty of the $\delta$ is introduced by the uncertainty of $\left(\sigma_{\star}\right)^{-}$, instead of $\left(\sigma_{\star}\right)^{+}$ (see also Section 4.2), the outflow can still be well constrained. The matching condition then constrains the outflow by singling out the outflow solution that satisfies $\left(B_{\phi}\right)^{-}=\left(B_{\phi}\right)^{+}$.

\footnotetext{
c.f. Equation (35) gives $\left(\mathcal{E}^{r}\right)^{-}=\left(\mathcal{E}^{r}\right)^{+}$.
}

\section{FLOW ALONG A PARABOLIC FIELD LINE WITH A FINITE-DISTANCE FAST POINT}

\subsection{Model Setup}

In general, the field configuration should be consistently determined by solving the trans-field equation (i.e., the GradShafranov equation). The trans-field equation in cold limit involves the stream function $\Psi$, and the derivative of the conserves quantities, $d \Omega_{F} / d \Psi, d \eta / d \Psi, d E / d \psi, d L / d \psi$ (Nitta et al. 1991; Beskin \& Par'ev 1993). However, solving the trans-field equation analytically is very challenging and beyond the scope of this paper.

On the other hand, we are interested in the case where the fast point of the outflow is located at a finite distance. It is therefore essential to consider an additional modification on the original force-free field line due to the MHD flow. We leave a better consideration of field configuration for a future work, and adopt the streamline function in Beskin \& Nokhrina (2006) as the prescribed parabolic field

$$
\Psi=\Psi_{0}+\epsilon,
$$

where $\Psi_{0}$ is the the flat spacetime parabolic force-free field generated by the toroidal surface current distribution, $I=C / 4 \pi r\left(1+\Omega_{F} r^{2}\right)^{1 / 2}$, on equatorial plane (Blandford 1976; Lee \& Park 2004),

$$
\Psi_{0}=\frac{\pi C}{\Omega_{F}} \sinh ^{-1}\left(\Omega_{F} r(1-\cos \theta)\right)
$$

and

$$
\epsilon f=\epsilon \pi C \Omega_{F} r \sin \theta, \quad \epsilon \ll 1
$$

is the perturbation introduced by the MHD effect. The constant $C$ is assumed to be unity. Note that by the help of the relation $\sinh ^{-1}(x)=\ln \left(x+\sqrt{x^{2}+1}\right), \quad \Psi_{0} \quad$ is proportional to $r(1-\cos \theta)$, which is the same as the dominating term of the parabolic field in BZ77. ${ }^{6}$ In addition, $\nabla \cdot B=0$ is guaranteed. It is shown in Beskin \& Nokhrina (2006) that, although $\epsilon f / \Psi_{0} \ll 1$ on the (outer) fast surface, the perturbation method is not applicable beyond the fast point. As a result, we can only discuss the flow solution up to the outer fast point.

The following assumptions for a PFD GRMHD flow along a hole-threading field line are considered. First, we assume $r_{\star}=r_{s}$. The assumption of $r_{\star}=r_{s}$ and $\left.u_{p}\right|_{r_{\star}}=0$ ensures $u_{p}$ has a smooth transition from $u_{p}<0$ (inflow) to $u_{p}>0$ (outflow). Second, to guarantee the flow is PFD, we require $\sigma_{\star} \gg 1$ (see also section 3.4.1 of Fendt \& Greiner 2001 for an estimation) and $\epsilon \ll 1$. Furthermore, we assume $\epsilon$ is constant along field lines. The higher the value of $\sigma_{\star}$, the more magnetically dominated the flows are.

Among the parameter space we seek the parameter set $\left\{\Omega_{F}, \eta, E, L\right\}$, which gives a similar time-averaged GRMHD simulation result in M06 for comparison. We therefore focus on a spinning black hole with its dimensionless spin $a=0.9$

\footnotetext{
6 Due to a similar toroidal surface current distribution, $I=C / 4 \pi r$ on the equatorial plane, the parabolic force-free field around a black hole considered in BZ77, $\Psi=\frac{C}{2}\{r(1-\cos \theta)+2(1+\cos \theta)[1-\ln (1+\cos \theta)]\}$, also follows $\Psi \propto r\left(1^{2}-\cos \theta\right)$ at large distance. This is one of the solutions of the source-free Maxwell equation in Schwarzschild spacetime, $\left(\frac{(1-2 / r)}{\sin \theta} \Psi_{, r}\right)_{, r}+\left(\frac{1}{r^{2} \sin \theta} \Psi_{, \theta}\right)_{\theta}=0$; while Equation (42) is one of the solutions of the source-free Maxwell equation in flat spacetime, $\left(\frac{1}{\sin \theta} \Psi_{, r}\right)_{, r}+\left(\frac{1}{r^{2} \sin \theta} \Psi_{, \theta}\right)_{, \theta}=0$.
} 


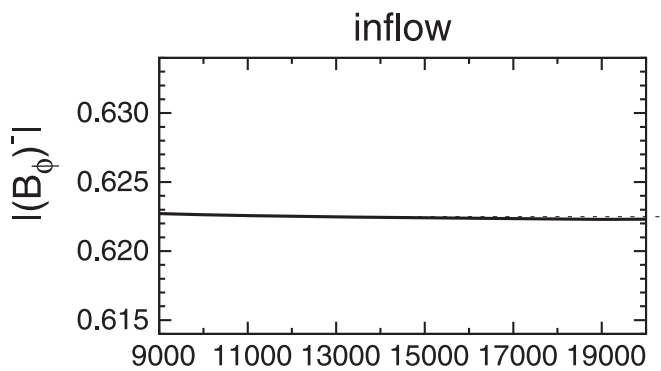

$\left(\sigma_{*}\right)^{-}$

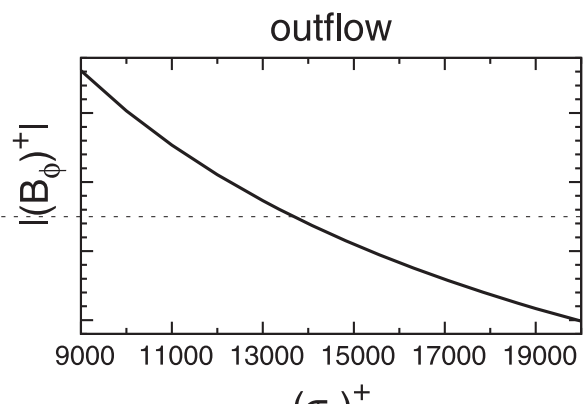

$\left(\sigma_{*}\right)^{+}$

Figure 2. Toroidal field, $B_{\phi}$, as a function of magnetization parameter, $\sigma_{\star}$, near the separation surface, $r_{s}$ for the inflow (left) and outflow (right) part of the solution. The superscript "-" and "+" denote the value computed at $r \rightarrow r_{s}^{-}$and $r \rightarrow r_{s}^{+}$, respectively. The matching conditions constrain the outflow by the inflow; that is, singling out the outflow solution that satisfies $\left(B_{\phi}\right)^{+}=\left(B_{\phi}\right)^{-}$(see Sections 3.2 and 4.2). When $\delta=1\left(\left(\sigma_{\star}\right)^{-}=\left(\sigma_{\star}\right)^{+} \simeq 13700\right)$, the matching condition is always satisfied.

and a field line that threads the event horizon at mid-latitude, $\theta=60^{\circ}$. As mentioned in Section 2.2, the set $\left\{\Omega_{F}, \eta, E, L\right\}$ can be equivalently determined by $\left\{\Omega_{F}, \sigma_{\star}, r_{\star}, r_{A}\right\}$. We adopt $r_{\star}=r_{s}$ (assumed) and $\Omega_{F}=1 / 2 \Omega_{H}$ (similar to the result of M06) in both inflow and outflow region. Then we determine $\sigma_{\star}$ and $r_{A}$ (note that once $r_{A}$ is determined, the location of the fast surface is determined accordingly) by the constraint (i) $\left(E_{\mathrm{EM}} / E_{\mathrm{FL}}\right) \sim 10^{2}$ near the separation surface similar to the case in M06, ${ }^{7}$ and (ii) the matching condition.

We note that $\Omega_{F} \approx 1 / 2 \Omega_{H}$ is self-consistently obtained in a steady PFD GRMHD flow solution for a monopole field geometry (Beskin \& Kuznetsova 2000), while it may not be relevant for a parabolic field geometry. BZ77 examined the parabolic streamline in which $\Omega_{F}$ decreases when shifting the angle from close to the pole to equatorial plan. In M06, the field geometry becomes almost monopolar in the vicinity of the horizon, so that $\Omega_{F} \approx 1 / 2 \Omega_{H}$ is observed along the field line (see also Beskin 2009). In the present paper, although the parabolic field is prescribed as a global field geometry, we nevertheless adopt the constant value of $1 / 2 \Omega_{H}$ in our fiducial solution for convenience.

\subsection{Matching the Inflow and Outflow}

Consistent solutions for PFD inflow and outflow along a field line are obtained iteratively until the matching condition is satisfied. For each $\sigma_{\star}$, because $\Omega_{F}\left(=\Omega_{H} / 2\right)$ and $r_{\star}$ (determined by where $K_{0}^{\prime}=0$ along the field line; see the Appendix) are known, we can solve WE (Equation (34)) for the flow solution by requiring the physical solution to pass through the fast surface. For example, for the case $\epsilon=0.065$, the profiles of $\left(B_{\phi}\right)^{-}$and $\left(B_{\phi}\right)^{+}$as a function of $\left(\sigma_{\star}\right)^{-}$and $\left(\sigma_{\star}\right)^{+}$, respectively, are shown in Figure 2.

A consistent inflow/outflow solution exists when a suitable set $\left(\epsilon,\left(\sigma_{\star}\right)^{-},\left(\sigma_{\star}\right)^{+}\right)$is applied. As mentioned in Section 3, the tendency is for $\left(B_{\phi}\right)^{-} \approx$ const. to result in multiple choices of $(\sigma)^{-}$such that $\left(B_{\phi}\right)^{-}=\left(B_{\phi}\right)^{+}$is satisfied. This leads to certain amount of freedom for choosing the value for $\delta$. For simplicity, $\delta=1$ is assumed, so $\left(\sigma_{\star}\right)^{-}=\left.\sigma_{\star}\right|^{+}=\sigma_{\star}$. We can read from Figure 2 that $\left(B_{\phi}\right)^{-}=\left(B_{\phi}\right)^{+}$is satisfied when $\left(\epsilon, \sigma_{\star}\right)=(0.065, \simeq 13700)$.

\footnotetext{
In top panel of Figure 7 of M06 $\Gamma_{\infty}^{(\mathrm{EM})} / \Gamma_{\infty}^{(\mathrm{MA})}$ is $\sim 10^{3}$ (see M06 for definitions), which is equivalent to $E_{\mathrm{EM}} / E_{\mathrm{FL}} \sim 10^{2}$ in terms of the definition in this paper. Note that our definition of $E_{\mathrm{EM}}$ is $4 \pi$ smaller than $\Gamma_{\infty}^{(\mathrm{EM})}$ used in M06. The factor of $4 \pi$ is absorbed into the definition of $F^{\mu \nu}$ in M06.
}

Table 1

Conserved Quantities of the Fiducial Flow Solutions

\begin{tabular}{|c|c|c|}
\hline \multicolumn{3}{|c|}{$\begin{array}{c}\mathrm{a}=0.9 \\
\left(\epsilon=0.065, \sigma_{\star} \simeq 13700\right)^{\mathrm{a}} \text { with } \delta=1\end{array}$} \\
\hline & Inflow & Outflow \\
\hline$|\eta|(\Psi) m$ & \multicolumn{2}{|c|}{$\simeq 7 \times 10^{-5}$} \\
\hline$E(\Psi) / \mu$ & $\simeq-112$ & $\simeq 114$ \\
\hline$L(\Psi) / \mu$ & $\simeq-720$ & $\simeq 724$ \\
\hline$\Omega_{F}(\Psi)$ & \multicolumn{2}{|c|}{$\simeq 0.157$} \\
\hline
\end{tabular}

Note.

${ }^{\mathrm{a}} \mathrm{A}$ consistent inflow/outflow solution is obtained when a suitable set $\left(\epsilon, \sigma_{\star}\right)$ is applied, such that the matching condition is satisfied (see Sections 3 and 4.2).

By the same method, for any specific value of $\epsilon$ (or $\left.\sigma_{\star}\right)$, there is a corresponding $\sigma_{\star}$ (or $\epsilon$ ) that satisfies the matching condition. The quantitative relation shows that as $\sigma_{\star}$ increases, $\epsilon$ also increases. This implies that, because there is more mass loading onto the field, the field progressively bunches up toward the rotational axis of the black hole. Finally, after $\sigma_{\star}$ is chosen by the matching condition, the parameter set $\left\{\Omega_{F}, \sigma_{\star}, r_{\star}, r_{A}\right\}$ of the inflow/outflow part of the solution is uniquely determined. The relaxation of the assumption $\delta=1$ is discussed at the end of Section 4.3.2.

\subsection{Self-Consistent Inflow/Outflow Solution}

\subsubsection{Flow Properties}

We adopt the parameter set $\left(\epsilon, \sigma_{\star}\right)=(0.065, \simeq 13700)$ as the fiducial model parameters, because the resulting flow solution satisfies our requirement $\left.\left(E_{\mathrm{EM}} / E_{\mathrm{FL}}\right)\right|_{r_{\star}} \sim 10^{2}$ (Section 4.1). The conserved quantities, $\left\{\Omega_{F}, \eta, E, L\right\}$, of our fiducial flow solution are shown in Table 1 . The mass loading $\eta$ changes sign according to $u^{r}$ and $\Psi_{, \theta}$ (Equation (10)) in inflow and outflow regions. Because the sign has no specific meaning, the absolute value $|\eta|$ is shown. By the assumption $\delta=1$, $\left|(\eta)_{\text {inflow }}\right|=\left|(\eta)_{\text {outflow }}\right|$ (Equation (39)).

In the inflow region $\left(u^{r}<0\right)$, both $E<0$ and $L<0$ indicate that the energy and angular momentum of the black hole is extracted outward $\left(\mathcal{E}^{r}>0\right.$ and $\left.\mathcal{L}^{r}>0\right) . E / \mu$ of the outflow gives the maximum possible value of the terminal Lorentz factor (Equation (20)). Although $\left(E_{\mathrm{EM}}\right)^{-}=\left(E_{\mathrm{EM}}\right)^{+}$under the assumption $\delta=1$, the absolution value of $E=E_{\mathrm{EM}}+E_{\mathrm{FL}}$ for 
Table 2

Properties of PFD GRMHD Flow Along the Same Hole-Threading Field Line

\begin{tabular}{lcc}
\hline \hline & Inflow Solution & Outflow Solution \\
\hline$u^{r}$ & $<0$ & $>0$ \\
$u^{\theta}$ & $>0$ & $<0$ \\
$u^{\phi}$ & $>0$ & $>0$ \\
$u^{t}$ & $>0$ & $>0$ \\
$E=E_{\mathrm{FL}}+\mathrm{E}_{\mathrm{EM}}$ & $<0$ & $>0$ \\
$E_{\mathrm{FL}}$ & $>0$ & $>0$ \\
$E_{\mathrm{EM}}$ & $<0$ & $>0$ \\
$L^{\prime}=L_{\mathrm{FL}}+\mathrm{L}_{\mathrm{EM}}$ & $<0$ & $>0$ \\
$L_{\mathrm{FL}}$ & $>0$ & $>0$ \\
$L_{\mathrm{EM}}$ & $<0$ & $>0$ \\
$\mathcal{E}^{r}=\mathcal{E}_{\mathrm{FL}}^{r}+\mathcal{E}_{\mathrm{EM}}^{r}$ & $>0$ & $>0$ \\
$\mathcal{E}_{\mathrm{FL}}^{r}$ & $<0$ & $>0$ \\
$\mathcal{E}_{\mathrm{EM}}^{r}$ & $>0$ & $>0$ \\
\hline
\end{tabular}

Note.

${ }^{\text {a }}$ For a stationary GRMHD inflow solution along a hole-threading field line, $\mathcal{E}$ ${ }_{\mathrm{FL}}^{r}<0$ is satisfied. In contrast, for an inflow along a non-hole-threading field line during transient phase, $\mathcal{E}_{\mathrm{FL}}^{r}>0$ is possible (e.g., Koide et al. 2002; Komissarov 2005).

the inflow is slightly smaller than the value for the outflow. This is because, in the inflow region, the fluid component $E_{\mathrm{FL}}$ (or $L_{\mathrm{FL}}$ ) has an opposite sign with the electromagnetic component $E_{\mathrm{EM}}$ (or $L_{\mathrm{EM}}$ ), partly canceling the electromagnetically extracted energy (or angular momentum); whereas in the outflow region, the fluid and electromagnetic components have the same sign, both carrying the energy and angular momentum outward. The general properties of different physical components for PFD inflows and outflows are provided in Table 2.

The extraction of black hole rotation energy by the GRMHD inflow is also indicated by the location of the inflow Alfvén surface. A remarkable feature in GRMHD is the existence of a negative energy region; once the Alfvén surface of an inflow resides inside such a region, the black hole energy is extracted outward (Takahashi et al. 1990). The inner boundary of the negative energy region is the inner light surface, and the outer boundary is defined by $g_{t t}+g_{t \phi} \Omega_{F}=0$. Thus, the region must be inside the ergosphere, where $g_{t t}>0$. As the flow becomes increasingly PFD, the location of the Alfvén surface moves toward the light surface, finally entering the negative energy region (see the Appendix). For PFD GRMHD inflow, the fast surface is located very close to, and almost coincides with, the black hole event horizon. This is why the PFD inflow solutions are all similar, as mentioned in Section 3. In Figure 3 we plot the locations of the Alfvén surface and fast surface of the flow, which share the same features mentioned previously.

\subsubsection{Radial Structure}

Let us now show the fiducial flow solution up to the fast surface in Figure 4, and compare the result (especially Figures 7 and 8) in M06. The top panel of Figure 4 shows the opening angle of the prescribed field, which roughly follows a single power law $\theta \propto r^{-0.52}$, which is in general more collimated compared to the result of M06. The locations of the characteristic surfaces overlap onto the profile. The Alfvén surfaces are located close to the light surfaces, and the inner fast surface is located close to the horizon. Note that in M06 the opening angle has different slope at a different radial range (see Figure 10 of M06). Instead, our prescribed field line follows a single power law. Nevertheless, with similar requirements at the separation surface $\left(\left.\left(E_{\mathrm{EM}} / E_{\mathrm{FL}}\right)\right|_{r_{\star}} \sim 10^{2}\right)$, the fast surface of the outflow is located at several hundred $r_{g}$ from the black hole, which is similar to the result of M06.

The second panel of Figure 4 shows the profiles of the electromagnetic energy component $E_{\mathrm{EM}}$ and the fluid energy component $E_{\mathrm{FL}}$ (both normalized by $\mu$ ), and the Lorentz factor $\Gamma$. Inside the ergosphere, $g_{t t}<0, \Gamma$ is ill-defined. Therefore, only the profile segments outside the ergosphere are plotted. At large distances, $g_{t t} \rightarrow-1, \Gamma \rightarrow-u_{t}=E_{\mathrm{FL}} / \mu$. In addition, for a $\mathrm{PFD}$ flow, $E \approx E_{\mathrm{EM}} \gg E_{\mathrm{FL}}$ when launching, so the maximum possible value of the terminal Lorentz factor, $\Gamma_{\infty}=E / \mu \approx E_{\mathrm{EM}} / \mu$ near the separation surface. As a result, the profile of $\Gamma$ along the streamline is therefore related to the conversion from $E_{\mathrm{EM}}$ to $E_{\mathrm{FL}}$. In the acceleration region $\left(\gtrsim 50 r_{g}\right), \Gamma$ roughly follows $\propto r^{0.6}$, which is similar to the result of M06, and the analytical result of $\Gamma \propto r^{0.5}$ obtained in Beskin $\&$ Nokhrina (2006). It is expected that a further acceleration takes place beyond the fast surface due to the magnetic nozzle effect (e.g., Camenzind 1989; Li et al. 1992). The conversion efficiency from Poynting to kinetic energy, which can be approximated by $\Gamma / \Gamma_{\infty}$, is closely related to the location of the fast surface. For example, when the fast surface is located at infinity, $\Gamma / \Gamma_{\infty} \approx 0$. For the outflow solution, $\Gamma / \Gamma_{\infty} \lesssim 0.1$ up to the fast surface, which is located at $\sim 300 r_{g}$. It is also interesting that the flow has already reached modest Lorentz factors $(\Gamma \sim 5)$ at the fast surface, and most of the Poynting energy has not yet been converted to kinetic energy. Note that, despite the final value of $\Gamma$ at the fast surface is similar to the result in M06, the Poynting energy in M06 at fast surface has already experienced a significant decay (more than one order of magnitude) up to the fast surface. The reason why the fluid energy is not correspondingly increasing may be due to dissipative processes. In the inner region beneath the separation surface, $-u_{t}=E_{\mathrm{FL}} / \mu \lesssim 1$, as expected because the fluid is strongly bounded by the black hole's gravity. In the outer region beyond the separation surface, $-u_{\mathrm{t}}>1$, which implies that the fluid is unbound and an outflow occurs.

Similar to the energy conversion between the fluid and the electromagnetic components, the increase of the fluid component of the angular momentum $L_{\mathrm{FL}}$ is at the expense of the electromagnetic component of the angular momentum $L_{\mathrm{EM}}$. The profiles of $L_{\mathrm{FL}}$ and $L_{\mathrm{EM}}$ (normalized by $\mu$ ) are shown in the third panel of Figure 4. Again, the profile of the fluid component $L_{\mathrm{FL}} / \mu=u_{\phi}$ is consistent with result of M06, but the decreases of the Poynting component in the simulation are much larger than our semi-analytical solution.

The radial and polar components of the four-velocity of the flow, $u^{r}$ and $u^{\theta}$, can be calculated from Equations (10) and (23), with $u_{p}$ determined by the WE. The other two components of the four-velocity, $u^{t}$ and $u^{\phi}$, can be obtained by solving

$$
-\mu\left(u_{t}+u_{\phi} \Omega_{F}\right)=E-\Omega_{F} L,
$$

subject to the normalization $u^{\alpha} u_{\alpha}=-1$. The velocity components $u^{r}$ and $u^{\theta}$ change signs across $r=r_{s}$, while the velocity components $u^{\phi}$ and $u^{t}$ remain positive in both the inflow and outflow regions. The angular velocity of the fluid, $\Omega=u^{\phi} / u^{t}$, 


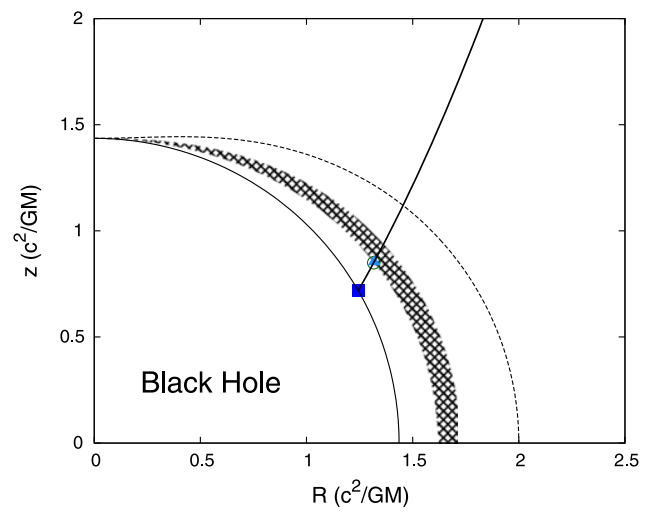

Figure 3. Characteristic points of a fiducial PFD GRMHD inflow. Toward the black hole: Alfvén surfaces (filled cyan triangles), light surfaces (empty green circles), and fast surfaces (filled blue squares). The event horizon and static limit (the outer boundary of the ergosphere) are shown by the thin solid and dashed lines, respectively. Field lines are represented by the thick solid line. The Alfvén surfaces are located inside the the negative energy region (shaded region), implying that the black hole energy is extracted outward.

which follows the black hole's rotation, is however always positive along the magnetic field line. At the separation surface, $u^{r}=u^{\theta}=0$, and hence $\Omega=\Omega_{F}$.

The radial and toroidal components of the orthonormal velocity at large distance are given by

$$
\begin{aligned}
\bar{u}^{r} & =\sqrt{g_{r r}} u^{r}, \\
\bar{u}^{\phi} & =\sqrt{g_{\phi \phi}} u^{\phi}
\end{aligned}
$$

as shown in the fourth panel of Figure 4. The profile of $\bar{u}^{r}$ is quite similar to the result in M06, but $\bar{u}^{\phi}$ has a relatively steeper profile compared to the simulation result. We suppose that this is related to the field configuration beyond the fast point, where we are not able discuss in current prescribed field configuration.

The orthonormal components of the magnetic fields at a large distance can be defined by

$$
\begin{aligned}
\bar{B}^{r} & =\sqrt{g_{r r}} B^{r}, \\
\bar{B}^{\phi} & =\sqrt{g_{\phi \phi}} B^{\phi} .
\end{aligned}
$$

Note that $\bar{B}^{r}$ is given initially when solving the WE, and $\bar{B}^{\phi}$, which is not initially known, can be determined after solving the WE. The bottom panel of Figure 4 shows the profile of the pitch angle, $\tan ^{-1}\left(\left|\bar{B}^{r} / \bar{B}^{\phi}\right|\right)$. Because $B^{r}$ and $B^{\phi}$ are both functions of $g_{t t}$ (see the Appendix), they quickly decrease and change sign when entering the ergosphere $\left(g_{t t}>0\right)$. As a result, $\left|\bar{B}^{r}\right|$ and $\left|\bar{B}^{\phi}\right|$ are ill-defined close to the black hole, and we only plot the profile in the region where $g_{t t}<0$. The reason why the pitch angle profile in M06 does not have this problem should be related to the definition of the field. The explicit form of the magnetic field we adopt is provided in the Appendix. Nevertheless, at far region (e.g., the outflow region), spacetime becomes more flat and the differences of the definition are less important, our result agrees with the result of M06. The locations where $\left|\bar{B}^{r}\right|=\left|\bar{B}^{\phi}\right|$ are close to the light surface. At a large distance, $\left|\bar{B}^{r}\right|$ is well-described by $\left|\bar{B}^{r}\right| \approx\left|\bar{B}^{\phi}\right| R_{L} / \sqrt{g_{\phi \phi}}$, where $R_{\mathrm{L}}=1 / \Omega_{F}$, as also obtained in M06.

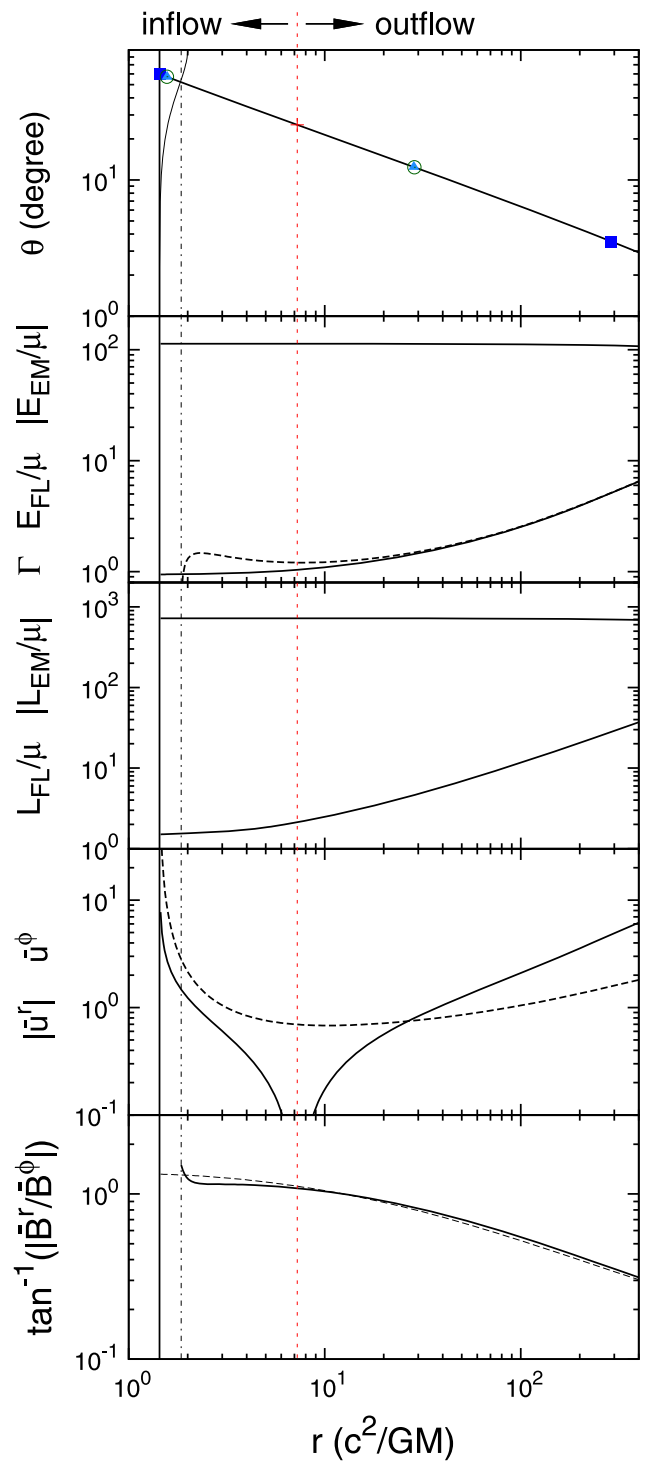

Figure 4. Fiducial PFD GRMHD flow solution properties along a field line. Top panel: jet opening angle of the prescribed field. Location of characteristic surfaces are also shown: separation point (plus sign), light surfaces (empty circles), Alfvén surfaces (filled triangles), and fast surfaces (filled squares). The thin vertical line indicates the angular profile of the static limit $\left(g_{t t}=0\right)$. Second panel: electromagnetic energy component (upper solid line), $E_{\mathrm{EM}}$, and fluid energy component (lower solid line), $E_{\mathrm{FL}}$, of the total energy $E=$ const. $=E_{\mathrm{EM}}+E_{\mathrm{FL}}$, in unit of fluid rest-mass energy. The profile of $\Gamma$ is shown only when $g_{t t}<0$ (dashed line). Third panel: electromagnetic (upper solid line), $L_{\mathrm{EM}}$, and fluid (lower solid line), $L_{\mathrm{FL}}$ components of total angular momentum, $L=$ const. $=L_{\mathrm{EM}}+L_{\mathrm{FL}}$. Fourth panel: the orthonormal velocities $\bar{u}^{r}$ and $\bar{u}^{\phi}$. Bottom panel: the pitch angle of the orthonormal field, $\tan ^{-1}\left(\left|\bar{B}^{r} / \bar{B}^{\phi}\right|\right)$ (solid line), which is well-described by $\tan ^{-1}\left(\left|R_{L} / \sqrt{g_{\phi \phi}}\right|\right)$ (dashed line) at large distance, where $R_{L}=1 / \Omega_{F}$. Because the orthonomal field is related to $g_{t t}$ and becomes ill-defined near the black hole, the pitch angle is only shown when $g_{t t}<0$. Along the field line, the location of the event horizon, the static limit, and the separation point are indicated by the vertical solid, dotted-dashed, and dashed lines, respectively.

At the end of this section, we discuss how the flow solution would change if we adopt a $\delta$, which also satisfies the matching condition, but does not equal to unity. Keep in mind that the outflow solution is well constrained by the matching condition, and the uncertainty of $\delta$ is due to the degeneracy of the inflow solutions (Section 3). As a result, the outflow solution will remain the same if a different value of $\delta$ is adopted. For the 

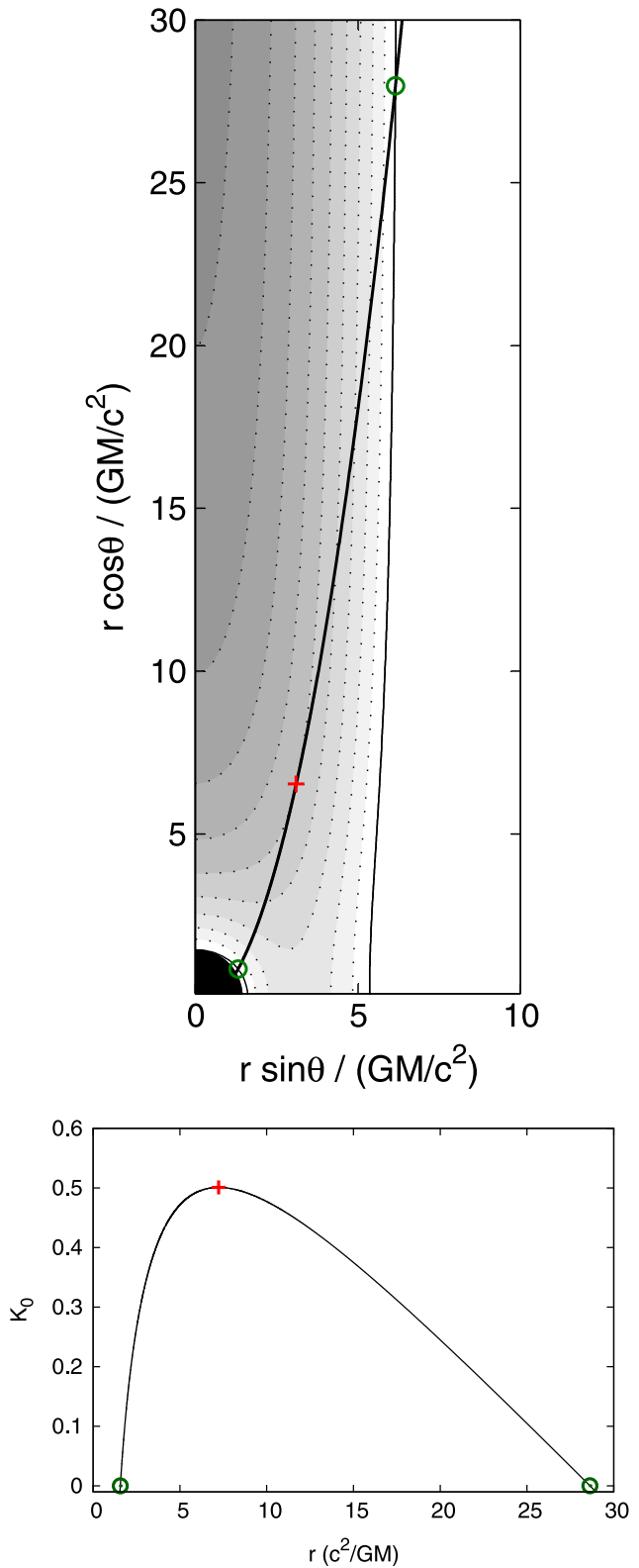

Figure 5. Determining the location of the light surfaces and the separation surface for a field line. Top: contour plot of $K_{0}$ for the case $a=0.9$ and $\Omega_{F}=\Omega_{H} / 2$. The black area represents the region enclosed by the black hole event horizon. The contour of $K_{0}=0$ is indicated by the solid line and the contours in the region $K_{0}>0$ are indicated by the dotted lines, with values at $K_{0}=0.1,0.2, \ldots, 0.8,0.9$. The thick solid line is a representative large-scale, black hole-threading field line. The two green circles indicate the locations of the light surfaces, which correspond to $K_{0}=0$, and the red cross indicates the location of the the separation surface at which $K_{0}^{\prime}=0$. Bottom: the value of $K_{0}$ along the field line. The locations of the light surfaces and separation surface are indicated by the same symbols.

PFD GRMHD flow, because the location of the Alfvén surface is always located near the inner light surface and the fast surface is always located close to the horizon, the flow dynamics will therefore be similar. That is, $u^{r}, u^{\theta}, u^{\phi}, u^{t}$, and therefore $E_{\mathrm{FL}} / \mu=-u_{t}$ and $L_{\mathrm{FL}} / \mu=u_{\phi}$ will remain almost unchanged. In addition, $B^{r}$ (prescribed) and $B^{\phi}$ (constrained by the Znajek's condition on horizon described in Section 3) will also remain similar. The electromagnetic component, $E_{\mathrm{EM}}$ and $L_{\mathrm{EM}}$, due to the dependence of $\propto 1 / \eta$, follow $\mid\left(E_{\mathrm{EM}}\right)_{\text {inflow }} /$
$\left(E_{\mathrm{EM}}\right)_{\text {outflow }}|=|\left(L_{\mathrm{EM}}\right)_{\text {inflow }} /\left(L_{\mathrm{EM}}\right)_{\text {outflow }}|=|(\eta)_{\text {outflow }} /$

$(\eta)_{\text {inflow }} \mid=1 / \delta$.

\section{SUMMARY}

A semi-analytical scheme is presented to investigate the cold, PFD GRMHD flow solution along a Kerr black holethreading field. The continuity of the outward Poynting energy flux across the separation surface is used as the matching condition to connect the inflow and outflow parts of a PFD GRMHD flow solution. We consider the parabolic field line of Beskin \& Nokhrina (2006), and therefore the resulting flow passes through all the critical points at a finite distance.

With similar black hole spin, angular velocity of the field, and magnetization at the separation surface, we are able to obtain a specific parameter set $\left\{\Omega_{F}, \sigma_{\star}, r_{\star}, r_{A}\right\}$ that gives inflow and outflow solutions in agreement with the time-averaged flow properties along a mid-level field line reported in the GRMHD simulation of M06.

In this current work, due to the limitation of the prescribed field configuration, we can only discuss the flow solution up to the outer fast surface.

Compared to the approaches of GRMHD and the general relativistic force-free electrodynamics (GRFFE; e.g., McKinney \& Narayan 2007) numerical simulation, the semi-analytical approach provides a complementary understanding of the relativistic jets, in the sense that the numerical dissipative process is absent, and the fluid component is included. The stationary solution obtained by the scheme can also be provided as a reference of the time-averaged GRMHD jet behavior in numerical simulations.

We thank the anonymous referee for helpful suggestions, which significantly improved the paper. We also thank C. Fendt for helpful information about GRMHD outflow solutions under cold limit, and Z. Younsi for proofreading. K.H. is supported by the Formosa Program between the National Science Council in Taiwan and Consejo Superior de Investigaciones Cientificas in Spain, administered through grant number NSC100-2923-M-007-001-MY3. Y.M. is supported by the Ministry of Science and Technology of Taiwan under the grant NSC 100-2112-M-007-022-MY3 and MOST 103-2112M-007-023-MY3, and by ERC Synergy Grant "BlackHoleCam: Imaging the Event Horizon of Black Holes."

\section{APPENDIX A \\ NOTES ON THE MAGNETIC FIELD}

Here we present the explicit form of the magnetic field. The covariant magnetic field defined in Equation (13)

$$
B_{\mu} \equiv \frac{1}{2} \epsilon_{\nu \mu \alpha \beta} F^{\alpha \beta} \xi^{\nu}
$$

can be alternatively written as

$$
B^{\mu} \equiv \frac{1}{2} \epsilon^{\nu \mu \alpha \beta} F_{\alpha \beta} \xi_{\nu},
$$

where $\epsilon_{\nu \mu \alpha \beta}=\sqrt{-g}[\nu \mu \alpha \beta]$, and $\epsilon^{\nu \mu \alpha \beta}=-\frac{1}{\sqrt{-g}}[\nu \mu \alpha \beta]$, with $\sqrt{-g}=\Sigma \sin \theta . \quad$ Because $\quad \xi^{\nu}=(1,0,0,0) \quad$ and $\xi_{\nu}=\left(g_{t t}, 0,0, g_{t \phi}\right)$, we can quickly read from the above definitions that $B_{t}=0$, but $B^{t} \neq 0$. 
The components of the magnetic field are therefore given by

$$
\begin{gathered}
B_{r}=\sqrt{-g} F^{\theta \phi}, \\
B^{r}=-\frac{g_{t t}+\Omega_{F} g_{t \phi}}{\sqrt{-g}} F_{\theta \phi}, \\
B_{\theta}=-\sqrt{-g} F^{r \phi}, \\
B^{\theta}=\frac{g_{t t}+\Omega_{F} g_{t \phi}}{\sqrt{-g}} F_{r \phi},
\end{gathered}
$$

and

$$
\begin{gathered}
B_{\phi}=\sqrt{-g} F^{r \theta}, \\
B^{\phi}=-\frac{g_{t t}}{\sqrt{-g}} F_{r \theta} .
\end{gathered}
$$

With the relations

$$
\begin{gathered}
F^{\theta \phi}=-g^{\theta \theta} \frac{g_{t t}+\Omega_{F} g_{t \phi}}{\Delta \sin ^{2} \theta} F_{\theta \phi}, \\
F^{r \phi}=-g^{r r} \frac{g_{t t}+\Omega_{F} g_{t \phi}}{\Delta \sin ^{2} \theta} F_{r \phi}, \\
F^{r \theta}=g^{r r} g^{\theta \theta} F_{r \theta},
\end{gathered}
$$

one can check $B^{r}=g^{r r} B_{r}, B^{\theta}=g^{\theta \theta} B_{\theta}$, and $B^{\phi}=g^{\phi \phi} B_{\phi}$. Note that, although $F_{\mu \nu}$ is finite at all regions, $B^{\mu}$ is ill-defined near a Kerr black hole because $g_{t t}$ changes sign when entering the ergosphere.

At large distance, the metric becomes Minkowski spacetime in spherical coordinates,

$$
d s^{2}=-d t^{2}+d r^{2}+r^{2} d \theta^{2}+r^{2} \sin ^{2} \theta d \phi^{2},
$$

and $\sqrt{-g}=r^{2} \sin \theta$. In this limit, the orthonormal field has the form

$$
\begin{gathered}
\bar{B}^{r} \equiv \sqrt{g_{r r}} B^{r}=\frac{1}{r^{2} \sin \theta} F_{\theta \phi}, \\
\bar{B}^{\theta} \equiv \sqrt{g_{\theta \theta}} B^{\theta}=-\frac{1}{r \sin \theta} F_{r \phi}, \\
\bar{B}^{\phi} \equiv \sqrt{g_{\phi \phi}} B^{\phi}=\frac{1}{r} F_{r \theta} .
\end{gathered}
$$

\section{APPENDIX B \\ CHARACTERISTIC SURFACES}

In the following we outline the characteristic surfaces of cold GRMHD flow, including the light surfaces, the separation surface, and the Alfvén and fast surfaces.

\section{B.1 Light Surfaces}

The surfaces defined by $K_{0}=0$ are the light surfaces. There are two light surfaces in a black-hole magnetosphere: the outer and the inner light surfaces. In the regions outside the light surfaces (where $K_{0}<0$ ) the fluid streams radially to avoid the toroidal velocity exceeding the speed of light. The outer light surface is formed in the same manner as the light cylinder in a pulsar magnetosphere, but it does not necessarily have a cylindrical shape in a Kerr spacetime. The inner light surface is formed due to strong gravity. Only when the black hole and the field line are not rotating does the inner light surface coincide with the black hole event horizon.

\section{B.2 Separation Surface}

In the cold limit the fluid acceleration along a field line, $u_{p}^{\prime}$ (where prime denotes the derivative along the flow streamline), changes direction at a certain point. The location, $r_{\mathrm{s}}$, at which the change occur, forms a separation surface (Takahashi et al. 1990; Hirotani et al. 1992). The fluid, starting with negligible velocity at $r_{\mathrm{s}}$, is accelerated inward inside the separation surface, creating an inflow. It is however accelerated outward outside the surface and develops an outflow.

The separation surface is inside the region bounded between the two light surfaces, and is determined via searching for where $K_{0}^{\prime}=0$ along each flow streamline in the calculations. Figure 5 shows how $r_{\mathrm{s}}$ on a specific field line (flow streamline) is determined in the demonstrative case with $K_{0}\left(a, \Omega_{F}\right)=K_{0}\left(0.9, \Omega_{H} / 2\right)$, (where $\Omega_{H}$ is the angular velocity of the black hole and $r_{+}$is the radius of the outer event horizon). The location where $K_{0}=0$ and $K_{0}^{\prime}=0$ along the field line can be read from the contours of $K_{0}$, which are part of the light surfaces and the separation surface, respectively. Note that the locations of the light surfaces and the separation surfaces are independent of the flow parameters, such as the mass loading, because they are determined only by $K_{0}\left(a, \Omega_{F}\right)$ and its derivative, $K_{0}^{\prime}$.

\section{B.3 Critical Surfaces for Cold GRMHD Flows}

Critical points appear when $D$ vanishes in the expression of $\left(\ln u_{p}\right)^{\prime}=N / D$. In the cold limit, there are two critical points. The Alfvén critical point corresponds to where $u_{p}$ is equal to the poloidal Alfvén speed, i.e.,

$$
u_{\mathrm{AW}}^{2}(r ; \Psi)=\frac{B_{p}^{2}}{4 \pi \mu n} K_{0},
$$

and the fast magnetosonic critical point corresponds to where $u_{p}$ equals the fast magnetosonic speed, i.e.,

$$
u_{\mathrm{FM}}^{2}(r ; \Psi)=u_{\mathrm{AW}}^{2}+\frac{B_{\phi}^{2}}{4 \pi \mu n \Delta \sin ^{2} \theta}
$$

(see Takahashi et al. 1990).

At the Alfvén surface

$$
M_{A}^{2}=\left.K_{0}\right|_{r_{A}} .
$$

Setting $u_{p}^{2}=u_{\mathrm{AW}}^{2}$ yields

$$
\frac{n}{4 \pi \mu} K_{0}=\eta^{2}
$$

Because $n$ and $\eta$ are positive, $K_{0}>0$ at the Alfvén surface. The Alfvén surfaces are therefore constrained inside the region bounded by the light surfaces (where $K_{0}=0$ ). In addition, $K_{0} \rightarrow 0$ as $\eta \rightarrow 0$, implying that the Alfvén surfaces approach the light surfaces when mass loading decreases.

Since the flow must be super-Alfvénic outside the light surfaces (when shocks are absent), would the flows downstream, outside the light surfaces eventually reach fast 
magnetosonic speeds? The answer is different for inflows and outflows. For the inflow, the magnetosonic speed is certainly reached, as causality requires that the flow speed must surpass all the possible characteristic speeds before the flow would enter the black hole event horizon (Takahashi et al. 1990). For the outflow, whether or not the flow speed will reach the fast magnetosonic speed depends on how fast the field decays along the flow (Takahashi \& Shibata 1998).

If the fast surface exists, the physical flow solution for the WE can be uniquely determined after specifying three of the conserved quantities, and searching for the last one until the the flow can smoothly pass the fast surface. (see, e.g., Appendix C in $\mathrm{Pu}$ et al. (2012) for the case of inflow as a demonstration). At the fast surface, where $u_{p}^{2}=u_{\mathrm{FM}}^{2}$, we have

$$
\frac{n}{4 \pi \mu}\left(K_{0}+\frac{B_{\phi}^{2}}{B_{p}^{2} \Delta \sin ^{2} \theta}\right)=\eta^{2} .
$$

By Equation (10), all else being equal, a relatively smaller $\eta$ is expected to produce a stronger $B_{\phi}\left(\propto F_{r \theta}\right)$ (see Pudritz et al. 2006 for a Newtonian version of this MHD feature). As a result, a smaller $K_{0}$ is required to satisfy Equation (B5) when a smaller mass loading is applied. That is, the location of the fast surface moves farther away from the light surface as the mass loading decreases. For a GRMHD inflow, the location of the fast surface gets closer to the event horizon.

\section{REFERENCES}

Asada, K., \& Nakamura, M. 2012, ApJL, 745, L28

Beckwith, K., Hawley, J. F., \& Krolik, J. H. 2008, ApJ, 678, 1180

Begelman, M. C., \& Li, Z.-Y. 1994, ApJ, 426, 269

Beskin, V. S., \& Par'ev, V. I. 1993, PhyU, 36, 529

Beskin, V. S., \& Kuznetsova, I. V. 2000, NCimB, 115, 795

Beskin, V. S., \& Nokhrina, E. E. 2006, MNRAS, 367, 375

Beskin, V. S. 2009, in MHD Flows in Compact Astrophysical Objects: Accretion in Winds and Jets, ed. V. S. Beskin (Berlin: Springer) Blandford, R. D. 1976, MNRAS, 176, 465

Blandford, R. D., \& Znajek, R. L. 1977, MNRAS, 179, 433 (BZ77)

Camenzind, M. 1986a, A\&A, 156, 137

Camenzind, M. 1986b, A\&A, 162, 32

Camenzind, M. 1987, A\&A, 184, 341
Camenzind, M. 1989, in Accretion Disks and Magnetic Fields in Astrophysics Vol. 156, ed. G. Belvedere (Kluwer: Cordrecht), 129

Cohen, M. H., Lister, M. L., Homan, D. C., et al. 2007, ApJ, 658, 232

Corbel, S., Fender, R. P., Tzioumis, A. K., et al. 2002, Sci, 298, 196

Fender, R., Wu, K., Johnston, H., et al. 2004, Natur, 427, 222

Fendt, C., \& Greiner, J. 2001, A\&A, 369, 308

Fendt, C., \& Camenzind, M. 1996, A\&A, 313, 591

Fendt, C., \& Ouyed, R. 2004, ApJ, 608, 378

Globus, N., \& Levinson, A. 2013, PhRvD, 88, 084046

Krishna, G., Witta, P. J., \& Dhurde, S. 2006, MNRAS, 369, 1287

Gu, M., Cao, X., \& Jiang, D. R. 2009, MNRAS, 396, 984

Hawley, J. F., \& Krolik, J. H. 2006, ApJ, 641, 103

Hirotani, K., Takahashi, M., Nitta, S.-Y., \& Tomimatsu, A. 1992, ApJ, 386,455

Hovatta, T., Valtaoja, E., Tornikoski, M., \& Lähteenmäki, A. 2009, A\&A, 494, 527

Jorstad, S. G., Marscher, A. P., Lister, M. L., et al. 2005, AJ, 130, 1418

Koide, S., Shibata, K., \& Kudoh, T. 1998, ApJ, 495, 63

Koide, S., Meier, D. L., Shibata, K., \& Kudoh, T. 2000, ApJ, 536, 668

Koide, S., Shibata, K., Kudoh, T., \& Meier, D. L. 2002, Sci, 295, 1688

Koide, S., \& Baba, T. 2014, ApJ, 792, 88

Komissarov, S. S. 2005, MNRAS, 359, 801

Lee, H. K., \& Park, J. 2004, PhRvD, 70, 063001

Li, Z.-Y., Chiueh, T., \& Begelman, M. C. 1992, ApJ, 394, 459

Lister, M. L., Aller, M. F., Aller, H. D., et al. 2013, AJ, 146, 120

Lyubarsky, Y. E. 2010, MNRAS, 402, 353

Lyutikov, M. 2011, MNRAS, 411, 422

McKinney, J. C., \& Gammie, C. F. 2004, ApJ, 611, 977

McKinney, J. C. 2006, MNRAS, 368, 1561 (M06)

McKinney, J. C., \& Narayan, R. 2007, MNRAS, 375, 531

Meier, D. L., Koide, S., \& Uchida, Y. 2001, Sci, 291, 84

Miller-Jones, J. C. A., Fender, R. P., \& Nakar, E. 2006, MNRAS, 367, 1432

Michel, F. C. 1969, ApJ, 158, 727

Mizuno, Y., Yamada, S., Koide, S., \& Shibata, K. 2004, ApJ, 615, 389

Nitta, S.-Y., Takahashi, M., \& Tomimatsu, A. 1991, PhRvD, 44, 2295

Phinney, E. S. 1983, PhD thesis, Univ. Cambridge

Pu, H.-Y., Hirotani, K., \& Chang, H.-K. 2012, ApJ, 758, 113

Pudritz, R. E., Rogers, C. S., \& Ouyed, R. 2006, MNRAS, 365, 1131

Pushkarev, A. B., Kovalev, Y. Y., Lister, M. L., \& Savolainen, T. 2009, A\&A, 507, L33

Sądowski, A., \& Sikora, M. 2010, A\&A, 517, A18

Semenov, V., Dyadechkin, S., \& Punsly, B. 2004, Sci, 305, 978

Takahashi, M., Nitta, S., Tatematsu, Y., \& Tomimatsu, A. 1990, ApJ, 363, 206

Takahashi, M., \& Shibata, S. 1998, PASJ, 50, 271

Tchekhovskoy, A., Narayan, R., \& McKinney, J. C. 2010, ApJ, 711, 50

Tchekhovskoy, A., Narayan, R., \& McKinney, J. C. 2011, MNRAS, 418, L79

Tchekhovskoy, A., \& McKinney, J. C. 2012, MNRAS, 423, L55

Znajek, R. L. 1977, MNRAS, 179, 457 